\title{
Terrestrial Applications of NASA Space Telerobotics Technologies
}

\author{
Dave Lavery \\ NASA Headquarters
}

\section{Introduction}

In 1985 the National Aeronautics and Space Administration(NASA)instituted a research program in telerobotics to develop and provide the technology for applications of telerobotics to the United States space program. The activities of the program are intended to most effectively utilize limited astronaut time by facilitating tasks such as inspection, assembly, repair, and servicing, as well as providing extended capability for remotely conducting planetary surface operations. As the program matured, it also developed a strong heritage of working with government and industry to directly transfer the developed technology into industrial applications.

\section{Program focus on user missions}

Sinceits inception, the Telerobotics Program currently conducted by the Office of Advanced Concepts and Technology (OACT) has been closely coordinated with the NASA organizations which are the intended recipients of the developed telerobotics technology. This coordination takes place at multiple levels, with the potential user community technology needs expressed both formally and informally to OACT.

At the highest strategic level, OACT works with the user offices and industry to develop an annual Integrated Technology Plan (ITP) in support of the civil space program. The purpose of the ITP is to serve as a strategic plan for the OACT space research and technology programs, and as a strategic planning framework for other NASA and industry participants in advocating and conducting technology developments. The integration of strategic requirements, directions and goals for the Space Telerobotics Program is incorporated within the ITP process. The ITP is revised annually to reflect changes in mission planning, approval of new focussed and research base efforts, and progress in ongoing technology development efforts.

In addition to the formal submission of requirements from the user program offices to OACT via the ITP process, each user organization works informally with the Telerobotics Program at a more detailed level to transmitrequirements to, and receive technology products from the program. This also includes gathering of requirements and opportunities from the terrestrial robotics industry, through an Industry Advisory Workshop held each year.

As these updated technology requirements are passed to the Telerobotics Program each year, the program is reassessed to determine the correlation between the requirements and the planned developments of the program. If appropriate, new tasks are initiated in the program to address new technology needs, or existing tasks may be re-targeted. At any given time, approximately $70 \%$ of the tasks within the program are targeted to address specific user requirements aligned with a specific planned mission (this is the "technology pull" portion of the program). The remaining $30 \%$ of the program is composed of tasks 
which address new innovative technologies. These technologies have been identified by the program as having a potential to significantly advance the state of the art, and worth investigating without a preidentified user requirement (this is the "technology push" portion of the program).

The anticipated robotics requirements forwarded by the user offices to the Telerobotics Program during this past year are summarized in Figure 1.

In previous years, these tasks were organized within the program by technology subdiscipline, such as supervisory control, operator interface, planning and control, perception, etc. This organization was useful to the program participants and robotics community to ease understanding of the component technologies being developed.
However, this organization made it difficult to identify how the tasks related to user needs. To resolve this situation the Telerobotics Program has been reorganized during the last year to better reflect the connections between the program tasks and the classes of planned user missions.

The Telerobotics Program has been restructured into three specific mission or application areas: on-orbit assembly and servicing, science payload tending, and planetary surface robotics. Within each of these areas, the program supports the development of robotic component technologies, development of complete robots, and implementation of complete robotic systems focussed on the specific mission needs. These three segments align with the application of space telerobotics to the class of missions identified by the

\begin{tabular}{|c|c|c|c|}
\hline \multicolumn{2}{|r|}{ Space Science } & \multirow{2}{*}{$\begin{array}{l}\text { Space Flight/Space.Station: } \\
\text { - telerobotic control system } \\
\text { software } \\
\text { - sensing and sensor fusion } \\
\text { - simplified collision avoidance } \\
\text { and trajectory planning } \\
\text { - automated task planning and } \\
\text { sequencing }\end{array}$} & \multirow[b]{2}{*}{$\begin{array}{l}\text { Mission From Planet Eanth*. } \\
\text { - robotic vision and perception } \\
\text { systems } \\
\text { - advanced proximity sensing } \\
\text { systems } \\
\text { - advanced dexterous end- } \\
\text { effectors } \\
\text { - high-efficiency, long term } \\
\text { lubrication for actuators }\end{array}$} \\
\hline Requirements: & $\begin{array}{l}\text { - low mass and volume } \\
\text { - lanetary surface rovers } \\
\text { - with multi-day lifetime } \\
\text { - autonomous and semi- } \\
\text { autonomous operation } \\
\text { - improved system robustness } \\
\text { - reduced operator command } \\
\text { cycles } \\
\text { - improved sensing and } \\
\text { representation of state and } \\
\text { - worksite } \\
\text { - miniaturized sensing and } \\
\text { - simputing systems } \\
\text { for small mobile systems } \\
\text { - improved system dexterity and } \\
\text { contact motion control } \\
\text { - terrain mapping and matching }\end{array}$ & & \\
\hline $\begin{array}{l}\text { Applicable } \\
\text { Missions: }\end{array}$ & $\begin{array}{l}\text { - MESUR Pathfinder } \\
\text { - MESUR Network } \\
\text { - Mars Sample Return } \\
\text { - Venus Landed Systems } \\
\text { (Discovery) } \\
\text { - Advanced robot surface } \\
\text { systems }\end{array}$ & $\begin{array}{l}\text { - Space Station Freedom } \\
\text { maintenance } \\
\text { - Space Station Freedom } \\
\text { operations } \\
\text { - on-orbit vehicle assembly and } \\
\text { processing }\end{array}$ & $\begin{array}{l}\text { - Artemis } \\
\text { - First Lunar Outpost } \\
\text { - Mars exploration } \\
\text { - Permanently Manned Lunar } \\
\text { and Mars Missions }\end{array}$ \\
\hline Challenges: & $\begin{array}{l}\text { - physical contact with planetary } \\
\text { - urfaces } \\
\text { - uncertain knowledge of } \\
\text { operating environment } \\
\text { - long operational phases } \\
\text { - radical reduction of life-cycle } \\
\text { costs } \\
\text { - multiple concurrent missions } \\
\text { - very high data rate science } \\
\text { - payloads } \\
\text { high speed simulation of } \\
\text { complex systems }\end{array}$ & $\begin{array}{l}\text { - generalized solutions to 7- } \\
\text { degree-of-freedom motion } \\
\text { - multi-arm coordinated } \\
\text { cooperative control } \\
\text { - reduced on-orbit computational } \\
\text { capability } \\
\text { - computation or } \\
\text { communications-induced time } \\
\text { delays }\end{array}$ & $\begin{array}{l}\text { - unknown dust contamination } \\
\text { characteristics } \\
\text { - low mass and volume } \\
\text { constraints } \\
\text { - long-duration pre-deployment } \\
\text { storage } \\
\text { - low-to no-maintenance } \\
\text { operations }\end{array}$ \\
\hline
\end{tabular}

Figure 1: Current space robotics user requirements 
potential space robotics user community (as summarized in Figure 1).

Two additional program segments have been defined to support the three focus areas. The Robotics Technology segment develops component technologies which have been determined to be of potential benefit in addressing multiple needs of the known robotics requirements. These elements of the program are typically long lead-time items, which may take many years to fully develop and bring to an appropriate level of readiness. This portion of the current program includes such elements as fundamentally new robotic joint designs, exoskeleton systems, fundamental robotic control theory development, and widelyapplicable proximity sensor technology. The Terrestrial Robotics element of the program provides a mechanism for the application of developed technologies into terrestrial task environments. These tasks move the technologies developed in the other elements of the program from the laboratory setting into operational use, and take advantage of the relatively easy terrestrial access, well understood environments, and myriad problems to be solved to demonstrate the applicability of space telerobotics.

\section{Links to other robotics programs}

Throughout the life of the NASA Telerobotics Program, NASA has worked to build and maintain coordination with other government robotics programs, including those of the National Science Foundation (NSF) and the National Institute of Standards and Technology (NIST). These efforts include cooperative activities, collaborative research, and external transfer of NASA-developed robotics technology. These efforts have three purposes: to develop industrial applications of telerobotics technology, to apply telerobotics technology to terrestrial science and research efforts, and to strengthen intra-government coordination. Several of the activities are summarized below, beginning with the efforts targeting development of industrial applications of telerobotics technology:

- The Automated Manufacturing Research Program, conducted by NIST is investigating automation in factoryfloor settings, and the relative advantages of improved work cells against more capable manipulation systems. NASA participates in the annual program review conducted by NIST, and coordinates with NIST to transfer NASA-developed robotics workcell technology into this effort.

- In previous years, NIST and the NASA telerobotics efforts have cooperatively developed several new technologies and architectures for the control of robotic systems. For example, the NASREM robot control architecture was jointly developed by NASA and NIST as a precursor to the NASA Flight Telerobotic Servicer program. The architecture is now used as a standard architecture definition methodology by many NASA, NIST and industry projects. NASA has directly supported NIST in several of these cooperative activities, with annual funding for robotics research reaching up to $\$ 1$ million per year.

- The NASA and NSF robotics research programs have jointly co-sponsored the "Bilateral Exchanges on the Approaches to Robotics in the United States and Japan" conference, which conducted investigations into the methods, techniques and technologies used by government and industry to research and develop fundamental new robotics technologies. The outcome of this activity was publication of a manuscript which contrasted the approaches used 
in the United States and Japan, and which offered NASA and NSF insights into the content of the robotics development programs supported by MITI, NASDA, and several Japanese industries.

- The Advanced Research Projects Agency (ARPA) has selected the Langley Research Center robotics program as one of their technical agents in the area of robotics. Under this agreement, LaRC and DARPA cooperatively issue university research grants to sponsor the development of innovative new robotics technologies, as well as increase robotics educational expertise in the United States.

- The program has maintained close ties with the U.S. space robotics industrial community, and monitored industrial developments of potential applicability to the NASA space robotics and planetary rover research efforts. For example, the Martin-Marietta Corporation participates in the Telerobotics Intercenter Working Group, and in technical program reviews and assessments such as the Space Systems Technology Advisory Council. This coordination facilitates the transfer of NASA-developed technologies to the space robotics industry, and aids in the rapid application of these technologies to terrestrial manufacturing and automation problems.

- The program coordinates with several robotics industry advisory and technology interchange groups, to facilitate the transfer of NASAdeveloped technology to the industrial community and receive comments on the overall direction and focus of the program. One such group is the Space Automation and Robotics Technical
Committee (SARTC) of the American Institute of Aeronautics and Astronautics which meets three or four times annually with the charter of disseminating information about space automation and robotics and promoting the technology to industry, academia, and government. The SARTC is composed of industry representatives from the aerospace community, as well as government and academia.

Some of the efforts which target application of telerobotics technology to terrestrial science and industry efforts are listed below:

- Several programs sponsored by NSF both sponsor and utilize telerobotics and robotics technology research and development. In 1992 NASA and NSF cooperated in conducting the Mt. Erebus Explorer project, a project to deploy a robot into the interior of a volcano crater in the Antarctic. This project, conducted as part of the Telerobotics Program and the Antarctic Space Analog Program, demonstrated innovative new robotics technologies developed by NASA. It is anticipated that this project will spawn several new activities which may revolutionize volcanic sample collection and lead to significant new applications of robotics in terrestrial field science operations. This project is being continued with the United States Geological Survey, and will deploy the Dante robot to a volcano in Alaska in the summer of 1994.

- In addition to the involvement with the NSF Polar Programs Division (which cooperated with the Mt. Erebus Explorer project), NASA is currently negotiating with the NSF Oceans Division to investigate the potential for application of NASA-developed robotics technology 
to underwater science sampling operations. Of particular interest is the underwater Remotely Operated Vehicle (ROV) technology which NASA developed and demonstrated under the Antarctic sea ice with the cooperation of NSF in 1992. Additional negotiations are underway with the NSF Information, Robotics and Intelligent Systems Division to jointly sponsor robotics research and investigate opportunities for transfer of NASA-developed robotics technologies to NSF grantees and research programs.

- The robotics laboratories at the Jet Propulsion Laboratory have been working with Computer Motion, Inc. to develop technologies for applications where human ability to perform a task is limited by human dexterity and physical capabilities. One specific application has been in minimally invasive laproscopic surgery. This type of medical procedure makes use of remote cameras, known as laproscopes, which are typically held by an assistant to the surgeon during a procedure. The assistant has control of the surgeons field of view, and the surgeons performance is often limited by the efficiency of communication with the assistant. To address this problem, the project has developed the Automated Endoscopic System for Optimal Positioning (AESOP), a robotic assistant which holds the laproscope and is guided by the surgeon with a foot- and/or handcontrolled interface. Thus the surgeon is able to gain control of the viewfield by direct coordination between himself and a robotic assistant. ${ }^{1}$

- JPL has also worked with Cybernet Systems Corporation to develop the PER-Force hand controller which manipulates robots or objects by "feel". this small backdrivable robot is combined with advanced machine vision processing and enhanced computer generated visual/tactile force feedback cues to enable an enhanced interface for the use on hazardous environment operations. This system has been implemented with a goal of integrating it within the manufacturing environment and tasks which have no immediate solution with hard automation or changes in methodology or workcell design. One example application being developed is pick-andplace operations for automobile transmission packing. ${ }^{2}$

- As an offshoot of work sponsored by the program, the Stanford University Aerospace Robotics Laboratory and Real-Time Innovations, Inc. have developed ControlShell, a next generation CASE framework for realtime system software development. ControlShell includes many systembuilding tools, including a graphical flow editor, a component data requirement editor, a state-machine editor, a distributed data flow manager, an execution configuration manager, an object database and a dynamic binding facility. ControlShell is being used in several applications, including the control of free-flying robots, underwater autonomous vehicles, and cooperatingarm robotic systems. ${ }^{3}$

- NASA has teamed up with Limbs of Love and a group of medical and prosthetics specialists, prosthetics users, insurance industry representatives, and university researchers to identify research objectives in prosthetic limbs. As part of this effort, the NASA Johnson Space Center has been actively working with Rice University to improve dexterous hand design and to develop a 
method for myoelectric control of multifinger hands. In theory, myoelectric control of robotic hands will require little or no mechanical parts and will greatly reduce the bulk and weight usually found in dexterous robotic hand control devices. An improvement in myoelectric control of multifinger hands will also benefit prosthetics users. ${ }^{4}$

This list is not exhaustive, but is a representative cross-section of the type of activities onducted by the NASA Telerobotics Program and other government organizations. Additional efforts have extended this coordination to industrial telerobotics research programs, to aid in the transfer of government-developed technology to the U.S. commercial/industrial robotics community. These efforts use two mechanisms to transfer the technology developed by the program.

The first mechanism pairs NASA researchers and commercial developers together to develop space telerobotics technology which is based on commercially-available products. As the terrestrial systems are extended to address the needs of the space telerobotics program by the researchers, the commercial partners are able to identify markets and applications for dual-use implementations of the new technologies, and rapidly incorporate them into new product lines. An example of this is the development of the "phantom robotic control" technology developed by JPL under the Advanced Teleoperation project. This technology has been developed as an extension to the commercial Interactive Graphics Robot Instructional Program (IGRIP) software package from Deneb Robotics. JPL has worked with Deneb to smoothly integrate the extension into the IGRIP package, and negotiated a mechanism to provide this extension to Deneb for commercialization.
Deneb has identified a new need for this technology, beyond the original application of space telerobotics, and plans to incorporate the extension into their commercial product line.

The second mechanism pairs NASA researchers with commercial developers to work jointly on the application of space telerobotics technologies to terrestrial problems. The commercial partners bring existing terrestrial robotics systems and capabilities into the project, and work jointly with NASA researchers to improve these systems through the application of space telerobotics technology. An example of this is the Hazardous Materials Handling Robot (HAZBOT) project at JPL, which is being conducted with the partnership of Remotec, Inc, and which is addressing the problem of hazardous chemical spill incident identification and mitigation through the use of robotics. JPL and Remotec worked to apply technologies developed by the Telerobotics Program to improve the offthe-shelf Remotec "Andros" mobile robot to satisfy the unique needs of the HAZBOT project. Several of the specific techniques and mechanisms developed during this process have been delivered back to Remotec for incorporation within their commercial product line.

The program has similar interactions with other members of the U.S. industrial robotics community, such as Robotics Research and Oceaneering. The current program plans include expanding these efforts to include a larger percentage of the U.S. robotics industry.

\section{Summary}

NASA has put in place a comprehensive planning process which fully integrates the development of new technologies with stated user requirements and defined application 
areas. The ongoing space automation and robotics program is focused on responding to the needs and requirements of internal agency users, but also produces significant spin-off products which are passed on to other government and industrial users for terrestrial utilization. The program fully involves agency users, NASA field centers, industry and academia in both the development and end-use of the developed A\&R technologies.

- Neville Marzwell, Darrin Yecker and Yulun Wang, Force-Controllable MacroMicro Manipulator and its Application to Medical Robotics, Technology 2003, Anaheim CA, December 1993.

2 Neville Marzwell, Charles Jacobus, Thomas Peurach and Brian Mitchell, Use ofInteractive Computer Vision and Robot Hand Controllers for Enhancing Manugacturing Safety, Technology 2003, Anaheim CA, December 1993.

${ }^{3}$ Stanley Schneider, Vincent Chen and Gerardo Pardo-Castelote, ControlShell: A Real-Time Software Framework. Proceedings of the International Conference on Robotics and Automation, IEEE, May 1994.

${ }^{4}$ Clifford Hess, Larry Li, Kristin Farry and Ian Walker, Application of Dexterous Space Robotics Technology to Myoelectric Prostheses, Technology 2003, Anaheim CA, December 1993. 